\title{
A Green and Simple Protocol one-pot Biginelli Condensation using Dicalcium Phosphate as Reusable Catalyst
}

\author{
Zakaria Benzekri, ${ }_{1}^{1}$ Redoua Benhdidou, ${ }_{1}$ Sara Hamia, ${ }^{1}$ Houda Serrar, ${ }^{1}$ Said Boukhris, ${ }^{1 *}$ Brahim Sallek, ${ }^{2}$ \\ Amina Hassikou ${ }^{1}$ and Abdelaziz Souizi ${ }^{1}$ \\ 1 Laboratory of Organic Chemistry, Organometallic and Theoretical. Faculty of Sciences, Ibn Tofail University, BO 133, 14000 \\ Kenitra, Morocco \\ 2 Laboratory of Agricultural Resources and Process Engineering. F Faculty of Sciences, Ibn Tofaïl University, BO 133, 14000 \\ Kenitra, Morocco \\ Corresponding author e-mail: nsboukhris@yahoo.com \\ Tel.: +212 66310 1030; fax: +212 537329433 .
}

Received November 14 ${ }^{\text {th }}, 2016$; Accepted March 22 ${ }^{\text {nd }}, 2017$.

\begin{abstract}
Dicalcium phosphate dihydrate (DCPD) was used as a green and reusable catalyst in order to synthesized two important categories of heterocycles, 3,4-dihydropyrimidin-2-ones and 3,4-dihydropyrimidin-2-thiones. The advantages of our method are as follow: short reaction times, green process, reduced environmental hazards and high isolated yield of products.

Key words: Heterogeneous catalysis; Green process; Dicalcium phosphate; 3,4-Dihydropyrimidin-2-ones; 3,4-Dihydropyrimidin-2-thiones.
\end{abstract}

\section{Introduction}

Multicomponent reactions (MCRs) are of great importance in both organic and medicinal chemistry for various reasons [1]. They offer significant advantages compared to conventional synthesis. Thus, MCR condensations involve three or more compounds that react in a one-pot reaction to form a new product. The Biginelli reaction is one of the most important multicomponent reactions for the synthesis of dihydropyrimidinones, consisting in the acid catalyzed cyclocondensation reaction of an aldehyde, a $\beta$-ketoester and a urea (or thiourea) [2]. Over the past decade, dihydropyrimidin-2-ones (DHPMs) and derivatives have attracted considerable attention in organic and medicinal chemistry because of their pharmacological and therapeutic properties [3]. Some derivatives have emerged due to their potential antiviral, antitumor, antibacterial and anti-inflammatory activities [3-5]. More recently, functionalized DHPMs are considered potent calcium channel blockers [6], antihypertensive agents [7], adrenergic antagonists [8] and neuropeptide Y (NPY) antagonists [9]. The original Biginelli protocol for the DHMPs preparation consisted of heating a mixture of the three components (1 equiv of an aldehyde 1, 1 equiv of $\beta$-keto ester 2 , and 1.5 equiv of urea 3 ), in ethanol with a catalytic amount of $\mathrm{HCl}[2,4]$. This procedure leads in one-pot reaction to the desired DHMPs, but in low yields, particularly for substituted aromatic and aliphatic aldehydes [7].
Resumen. Se empleó el fosfato dicálcico dihidratado (DCPD) como catalizador verde y reusable para sintetizar dos importantes categorías de heterociclos, 3,4-dihidropirimidin-2-onas y 3,4-dihidropirimidin-2-tionas. Nuestro método presenta las siguientes ventajas: tiempos de reacción cortos, proceso verde, peligros ambientales reducidos y altos rendimientos de los productos aislados.

Palabras clave: Catalizador heterogéneo; Proceso verde; Fosfato dicálcico; 3,4-Dihidropirimidin-2-onas; 3,4-Dihidropirimidin-2-tionas.

The Biginelli condensation is also performed using Lewis acids such as $\mathrm{BF}_{3} \mathrm{OEt}_{2}$, polyphosphate esters, and reagents like $\mathrm{InCl}_{3}, \mathrm{Mn}(\mathrm{OAc})_{3}$, trimethylsilyltriflate, $\mathrm{LaCl}_{3} \cdot 7 \mathrm{H}_{2} \mathrm{O}, \mathrm{Ce}-$ $\mathrm{Cl}_{3} \cdot 7 \mathrm{H}_{2} \mathrm{O}, \mathrm{LiClO}_{4}, \mathrm{Yb}(\mathrm{OTf})_{3}, \mathrm{ZrCl}_{4}$, or $\mathrm{ZrOCl}_{2}$, among others [8,10-24], STO/Al-P [25]. $\mathrm{FeCl}_{3}$ /Nanopore silica [26], $\mathrm{PhB}(\mathrm{OH})_{2}$ [27], $\mathrm{Cu}\left(\mathrm{NH}_{2} \mathrm{SO}_{3}\right)_{2}$ [28], $\mathrm{Fe}_{3} \mathrm{O}_{4}$ nanoparticles [29], sulfonated carbon materials (SCMs) [30], N-acetyl glycine (NAG) [31-32], $\mathrm{Yb}(\mathrm{OTf})_{3}$ [33], 3-[(3-(trimethoxysilyl)propyl) thio]propane-1-oxy-sulfonic acid (TMSPTPOSA) [34], $\mathrm{Fe}(\mathrm{OTs})_{3} \cdot 6 \mathrm{H}_{2} \mathrm{O}$ [35], 3-(2-carboxybenzoyl)-1-methyl-1H-imidazolium chloride [Cbmim]Cl [36], Boehmite nanoparticles [37], $\mathrm{TiO}_{2}$-CNTs [38] and $\mathrm{H}_{5} \mathrm{PW}_{10} \mathrm{~V}_{2} \mathrm{O}_{40} / \mathrm{Pip}-\mathrm{SBA}-15$ [39]. However, many of these methods use longer reaction times, strong acidic conditions and stoichiometric amounts of catalysts.

In the present study, we report a green and valid procedure for the synthesis of 3,4-dihydro pyrimidin-2-ones via one-pot condensation of aromatic aldehydes, acetylacetone or ethyl acetoacetate and urea or thiourea in the presence of dicalcium phosphate dihydrate (DCPD) [40] as a heterogeneous catalyst.

\section{Results and Discussion}

Dicalcium phosphate dihydrate (DCPD) was synthesized by double decomposition of calcium nitrate tetrahydrate and 
ammonium dihydrogenophosphate. After $2 \mathrm{~h}$ of maturation, the suspension was filtered, washed and freeze dried. The appropriate physical methods which should be used to confirm the identity of the product are IR absorption spectroscopy, XRD, elemental analyses of calcium and phosphate concentrations, scanning electron microscopy, and specific surface by BET [40].

The choice of appropriate reaction conditions is important for a successful synthesis. To study the effect of catalyst on the reaction, the one-pot condensation of benzaldehyde 1a $(1 \mathrm{mmol})$, acetylacetone $2(1 \mathrm{mmol})$ and urea $3(1.5 \mathrm{mmol})$ was chosen as a model reaction in the presence of $5 \mathrm{~mol} \%$ dicalcium phosphate dihydrate (DCPD) at reflux conditions in $\mathrm{EtOH}$ (Scheme 1). The efficiency of the condensations was mainly affected by the amount of the catalyst. Biginelli reaction in the absence of catalyst (Table 1, entry 1) was found to be very slow and gave a poor yield of DHPM (39\% isolated) after a longer reaction time (45 $\mathrm{min}$ ), while good results were obtained in the presence of DCPD. The optimal amount of the catalyst was 7 mol\% (Table 1, entry 6), whereas a higher amount of the catalyst did not increase the yield noticeably (Table 1, entry 7).

In order to optimize the reaction conditions, various solvents such as $\mathrm{EtOH}, \mathrm{MeOH}$, butanol, isopropanol, $\mathrm{CH}_{3} \mathrm{CN}$, AcOEt, THF and solvent-free conditions in the presence of DCPD as the catalyst were used (Table 1). Reaction in $\mathrm{CH}_{3} \mathrm{CN}$ and AcOEt gave low product yields even after $35 \mathrm{~min}$ (Table 1, entries 11 and 13). The yields were moderate in case of methanol, butanol, isopropanol, THF and solvent-free condition. The best results were obtained when the reaction was carried out in ethanol at reflux for $35 \mathrm{~min}$ in the presence of $7 \mathrm{~mol} \%$ of catalyst (Table 1, entry 6).

After the condensation, the DCPD catalyst was filtered, washed with $\mathrm{EtOH}$ and dried in vacuum oven at $100{ }^{\circ} \mathrm{C}$. The activity of the recovered catalyst did not decrease appreciably even after six consecutive runs, and no significant loss of conversion was observed, depicting the high stability of the catalyst under the reaction conditions (Table 2).

The generality of reaction was studied under optimal conditions by varying the structure of aldehyde (Scheme 2 ). As the data in Table 3 show, the catalyst was highly efficient for the reaction and all aromatic aldehydes (containing electron-withdrawing substituents or electron-donating substituents) afforded the corresponding products in high yields within short reaction times.

The efficiency of our newly synthesized catalyst for the synthesis of 3,4-dihydropyrimidin-2-ones and 3,4-dihydro-
Table 1. Optimization of the reaction conditions. ${ }^{\mathrm{a}}$

\begin{tabular}{ccccc}
\hline Entry & $\begin{array}{c}\text { DCPD } \\
(\mathrm{mol} \%)\end{array}$ & $\begin{array}{c}\text { Solvent } \\
(10 \mathrm{~mL})\end{array}$ & Time $(\mathrm{min})$ & $\begin{array}{c}\text { Yield }^{\mathrm{b}} \\
(\%)\end{array}$ \\
\hline 1 & -- & EtOH & 45 & 39 \\
2 & 1 & EtOH & 35 & 67 \\
3 & 4 & EtOH & 35 & 91 \\
4 & 5 & EtOH & 35 & 91 \\
5 & 6 & EtOH & 35 & 92 \\
6 & 7 & EtOH & 25 & 98 \\
7 & 8 & EtOH & 35 & 97 \\
8 & 7 & MeOH & 35 & 64 \\
9 & 7 & Butanol & 35 & 65 \\
10 & 7 & Isopropanol & 35 & 63 \\
11 & 7 & CH ${ }_{3} \mathrm{CN}$ & 35 & 51 \\
12 & 7 & THF & 35 & 71 \\
13 & 7 & AcOEt & 35 & 60 \\
14 & 7 & Solvent-free & 35 & 64 \\
\hline
\end{tabular}

${ }^{a}$ Reaction conditions: benzaldehyde (1a) (1 mmol), acetylacetone (2a) (1 mmol), urea (3a) (1.5 mmol), DCPD, $10 \mathrm{~mL}$ solvent at reflux. ${ }^{\mathrm{b}}$ Isolated yield.

Table 2. Reusability of the catalyst in the synthesis of 3,4-dihydropyrimidin-2-one $\mathbf{4 a}$.

\begin{tabular}{cc}
\hline Run & ${\text { Yield }(\%)^{\mathrm{a}}}^{1}$ \\
2 & 98 \\
3 & 97 \\
4 & 96 \\
5 & 96 \\
6 & 94 \\
\hline
\end{tabular}

a Isolated yields.<smiles>O=Cc1ccccc1</smiles>

1

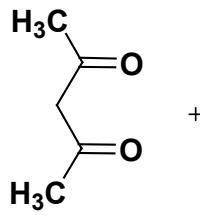

2<smiles>NC(N)=O</smiles>

3<smiles>CC(=O)C1=C(C)NC(=O)NC1c1ccccc1</smiles>

4a

Scheme 1. Synthesis of 3,4-dihydropyrimidin-2(1H)-one 4a catalyzed by dicalcium phosphate dihydrate (DCPD). 


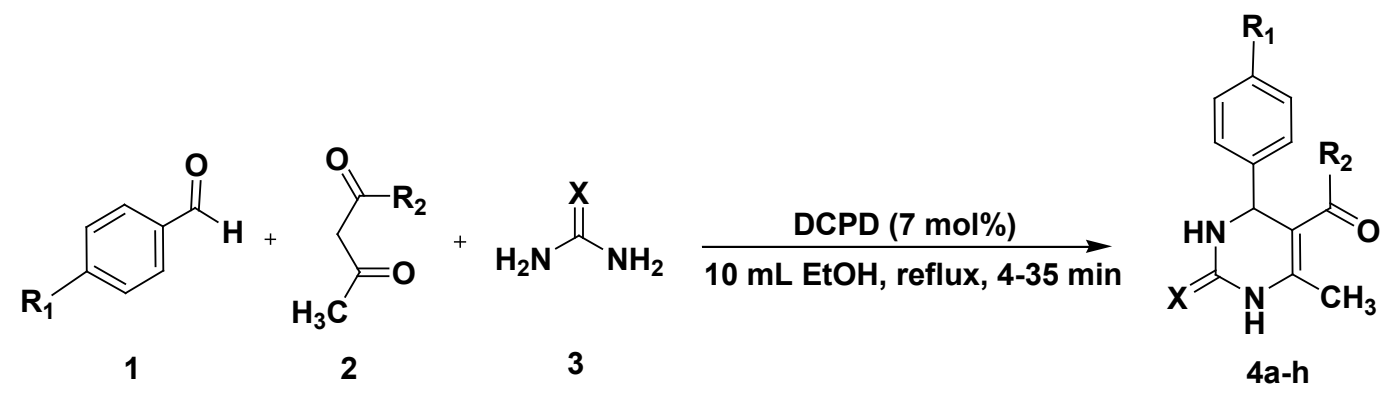

Scheme 2. Synthesis of 3,4-dihydropyrimidin-2(1H)-ones/thiones derivatives catalyzed by DCPD.

Table 3. Synthesis of pyrimidin-2-ones/thiones in the presence of DCPD catalyst.

\begin{tabular}{|c|c|c|c|c|c|}
\hline Entry & $\mathrm{R}_{1}$ & $\mathrm{R}_{2}$ & $X$ & Time (min) & Yield $\%$ \\
\hline $4 a$ & $\mathrm{H}$ & $\mathrm{Me}$ & $\mathrm{O}$ & 25 & 98 \\
\hline $4 \mathrm{~b}$ & $\mathrm{H}$ & OEt & $\mathrm{O}$ & 5 & 68 \\
\hline $4 d$ & $\mathrm{Cl}$ & OEt & $\mathrm{S}$ & 4 & 96 \\
\hline $4 \mathrm{e}$ & $\mathrm{NO}_{2}$ & OEt & $\mathrm{O}$ & 4 & 89 \\
\hline $4 \mathrm{~h}$ & $\mathrm{~N}(\mathrm{Me})_{2}$ & OEt & $\mathrm{O}$ & 5 & 97 \\
\hline
\end{tabular}

${ }^{\text {a }}$ Isolated yields.

Table 4. Comparison of the catalytic efficiency of DCPD with other catalysts in the preparation of 3,4-dihydropyrimidin-2-ones via Biginelli reaction.

\begin{tabular}{|c|c|c|c|c|c|}
\hline Entry & Catalyst & Solvent & Time & Yield $\%$ & Ref. \\
\hline 1 & Calix arene sulfonic Acid & Ethanol & $20-48 \mathrm{~min}$ & $46-93$ & {$[41]$} \\
\hline 2 & $\left(\mathrm{Al}_{2}\left(\mathrm{SO}_{4}\right)_{3}\right) \cdot 18 \mathrm{H}_{2} \mathrm{O}$ & PEG-400 & $3-150 \mathrm{~min}$ & $35-96$ & {$[42]$} \\
\hline 4 & $N, N$-diethyl- $N$-sulfo ethanammonium chloride & Ethanol & $15 \mathrm{~min}$ & 84 & {$[44]$} \\
\hline 5 & {$\left[\mathrm{Et}_{3} \mathrm{~N}-\mathrm{SO}_{3} \mathrm{H}\right] \mathrm{HSO}_{4}$} & Solvent-free & $15-100 \mathrm{~min}$ & $81-96$ & {$[45]$} \\
\hline
\end{tabular}

pyrimidin-2-thiones 4 was further evaluated by comparing its performance with several other catalysts reported in the literature [41-46]. The results revealed that DCPD catalyst performed much more effectively than others in terms of providing a very short reaction time, mild conditions and a high yield of the product (Table 4).

\section{Conclusions}

In summary, we have developed a convenient and highly efficient method for the synthesis of 3,4-dihydropyrimidin-2-ones and 3,4-dihydropyrimidin-2-thiones derivatives. The mild reaction conditions, experimental simplicity, straight forward purification procedures, excellent yields with short reaction times, as well as the application of green chemistry principles, are the advantages of this methodology.

\section{Experimental Section}

\section{General procedure for the synthesis of catalyst (DCPD)}

Dicalcium phosphate dihydrate (DCPD) was prepared by mixing $50 \mathrm{~mL}$ of a $0.3 \mathrm{M}$ solution of ammonium dihydrogenophosphate $\left(\left(\mathrm{NH}_{4}\right) \mathrm{H}_{2} \mathrm{PO}_{4}\right)$ with $50 \mathrm{~mL}$ of a $0.5 \mathrm{M}$ solution of calcium 
nitrate tetrahydrate $\left(\mathrm{Ca}\left(\mathrm{NO}_{3}\right)_{2} \cdot 4 \mathrm{H}_{2} \mathrm{O}\right)$ at room temperature. After $2 \mathrm{~h}$ of maturation, the precipitate was collected by vacuum filtration, washed with deionized water and freeze dried overnight.

\section{Typical experimental procedure for the synthesis of compounds 4a-h}

A mixture of urea or thiourea $(1.5 \mathrm{mmol})$, substituted benzaldehyde $(1 \mathrm{mmol}), \beta$-ketoester $(1 \mathrm{mmol})$ and dicalcium phosphate dihydrate (DCPD) $(7 \mathrm{~mol} \%)$ in ethanol $(10 \mathrm{ml})$ was heated to reflux for a specified time (TLC monitoring). Solid precipitated out from the reaction mixture was filtered, and recrystallized from methanol to afford pure 3,4-dihydropyrimidin-2(1H)-ones/ thiones as yellow/white solids. The catalyst was recovered by vacuum filtration during recrystallization and treated by the procedure mentioned below.

The products prepared 4 are known compounds [22-28] and were confirmed by comparing the ${ }^{1} \mathrm{H}$ NMR and ${ }^{13} \mathrm{C}$ NMR data with authentic samples reported in the literature.

5-Acetyl-6-methyl-4-phenyl-3,4-dihydropyrimidin-2-one (4a). Mp 237-239 ${ }^{\circ} \mathrm{C}$ (lit.[26] 238-239 $\left.{ }^{\circ} \mathrm{C}\right) .{ }^{1} \mathrm{H}$ NMR (300 MHz, DMSO-d $\left._{6}\right): \delta 9.16(\mathrm{~s}, 1 \mathrm{H}, \mathrm{NH}), 7.71(\mathrm{~s}, 1 \mathrm{H}, \mathrm{NH}), 7.20-7.30(\mathrm{~m}$, $5 \mathrm{H}, \mathrm{ArH}), 5.12(\mathrm{~d}, 1 \mathrm{H}, J=3 \mathrm{~Hz}, \mathrm{CH}), 2.22\left(\mathrm{~s}, 3 \mathrm{H}, \mathrm{CH}_{3}\right) ;{ }^{13} \mathrm{C}$ NMR $\left(75 \mathrm{MHz}\right.$, DMSO-d $\left._{6}\right): \delta 165.1,152.6,148.8,145.3$, $128.8,126.7,99.7,54.4,28.3,14.5$.

Ethyl 6-methyl-2-oxo-4-phenyl-1,2,3,4-tetrahydropyrimidine5-carboxylate (4b). $\mathrm{Mp} 208-210^{\circ} \mathrm{C}$ (lit.[23] 207-208 ${ }^{\circ} \mathrm{C}$ ). ${ }^{1} \mathrm{H}$ NMR (300 MHz, DMSO-d $): \delta 9.16(\mathrm{~s}, 1 \mathrm{H}, \mathrm{NH}), 7.71(\mathrm{~s}, 1 \mathrm{H}$, NH), 7.21-7.32 (m, 5H, ArH), 5.12 (s, 1H, CH), 3.97 (q, J=7.4 $\left.\mathrm{Hz}, 2 \mathrm{H}, \mathrm{OCH}_{2}\right), 2.22\left(\mathrm{~s}, 3 \mathrm{H}, \mathrm{CH}_{3}\right), 1.10$ (t, $J=7.4 \mathrm{~Hz}, 3 \mathrm{H}$, $\left.\mathrm{OCH}_{2} \mathrm{CH}_{3}\right) ;{ }^{13} \mathrm{C}$ NMR $\left(75 \mathrm{MHz}\right.$, DMSO-d $\left.\mathrm{d}_{6}\right): \delta 165.8,152.6$, 148.8, 145.4, 128.8, 127.7, 126.8, 99.7, 59.6 54.4, 18.2, 14.5.

Ethyl4-(4-chlorophenyl)-6-methyl-2-oxo-1,2,3,4-tetrahydropyrimidine-5-carboxylate (4c). Mp $212-214^{\circ} \mathrm{C}$ (lit.[22] 212$\left.214^{\circ} \mathrm{C}\right) .{ }^{1} \mathrm{H}$ NMR (300 MHz, DMSO-d 6 ): $\delta 9.28(\mathrm{~s}, 1 \mathrm{H}, \mathrm{NH})$, 7.73 (s, 1H, NH), 7.09-7.55 (m, 4H, ArH), $5.57(\mathrm{~s}, 1 \mathrm{H}, \mathrm{CH})$, $3.89\left(\mathrm{q}, J=7.3 \mathrm{~Hz}, 2 \mathrm{H}, \mathrm{OCH}_{2}\right), 2.27\left(\mathrm{~s}, 3 \mathrm{H}, \mathrm{CH}_{3}\right), 0.98(\mathrm{t}, J=$ $\left.7.3 \mathrm{~Hz}, 3 \mathrm{H}, \mathrm{OCH}_{2} \mathrm{CH}_{3}\right) ;{ }^{13} \mathrm{C}$ NMR $\left(75 \mathrm{MHz}, \mathrm{DMSO}_{6}\right): \delta$ $161.6,150.0,141.3,133.0,130.7,129.2,128.4,97,9,59.6$, $51.6,18.1,14,3$.

Ethyl 4-(4-chlorophenyl)-6-methyl-2-thioxo-1,2,3,4-tetrahydropyrimidine-5-carboxylate (4d). Mp $186-188^{\circ} \mathrm{C}$ (lit.[25] $\left.186-188^{\circ} \mathrm{C}\right) .{ }^{1} \mathrm{H}$ NMR $\left(300 \mathrm{MHz}\right.$, DMSO-d $\left.{ }_{6}\right): \delta 9.22(\mathrm{~s}, 1 \mathrm{H}$, $\mathrm{NH}), 7.75(\mathrm{~s}, 1 \mathrm{H}, \mathrm{NH}), 7.21-7.38(\mathrm{~m}, 4 \mathrm{H}, \mathrm{ArH}), 5.12(\mathrm{~s}, 1 \mathrm{H}$, $\mathrm{CH}), 3.97$ (q, $\left.J=7.2 \mathrm{~Hz}, 2 \mathrm{H}, \mathrm{OCH}_{2}\right), 2.22\left(\mathrm{~s}, 3 \mathrm{H}, \mathrm{CH}_{3}\right), 1.07$ (t, $\left.J=7.2 \mathrm{~Hz}, 3 \mathrm{H}, \mathrm{OCH}_{2} \mathrm{CH}_{3}\right) ;{ }^{13} \mathrm{C}$ NMR $(75 \mathrm{MHz}$, DMSO-d 6 ): $\delta$ 165.6, 152.4, 149.1, 144.2, 132.2, 128.8, 128.6, 99.3, 59.7, $53.8,18.2,14.5$.

Ethyl 6-methyl-4-(4-nitrophenyl)-2-oxo-1,2,3,4-tetrahydropyrimidine-5-carboxylate (4e). Mp $210-212^{\circ} \mathrm{C}$ (lit.[22]
211-212 $\left.{ }^{\circ} \mathrm{C}\right) .{ }^{1} \mathrm{H}$ NMR $\left(300 \mathrm{MHz}, \mathrm{DMSO}-\mathrm{d}_{6}\right): \delta 9.32(\mathrm{~s}, 1 \mathrm{H}$, $\mathrm{NH}), 8.20(\mathrm{~d}, J=9 \mathrm{~Hz}, 2 \mathrm{H}), 7.86(\mathrm{~s}, 1 \mathrm{H}, \mathrm{NH}), 7.47-7.56(\mathrm{~m}, 4 \mathrm{H}$, $\mathrm{ArH}), 5.26(\mathrm{~s}, 1 \mathrm{H}, \mathrm{CH}), 3.97\left(\mathrm{q}, J=7.4 \mathrm{~Hz}, 2 \mathrm{H}, \mathrm{OCH}_{2}\right), 2.24(\mathrm{~s}$, $\left.3 \mathrm{H}, \mathrm{CH}_{3}\right), 1.09\left(\mathrm{t}, J=7.4 \mathrm{~Hz}, 3 \mathrm{H}, \mathrm{OCH}_{2} \mathrm{CH}_{3}\right) ;{ }^{13} \mathrm{C}$ NMR $(75$ MHz, DMSO-d $\mathrm{d}_{6}$ ): $\delta 165.5,158.1,152.44,149.8,147.2,139.6$, $130.7,128.1,124.6,98.7,62.0,59.9,54.2,18.3,14.5$.

Ethyl 4-(4-methoxyphenyl)-6-methyl-2-oxo-1,2,3,4-tetrahydropyrimidine-5-carboxylate (4f). Mp 200-202 ${ }^{\circ} \mathrm{C}$ (lit.[27] 200-202 $\left.{ }^{\circ} \mathrm{C}\right) .{ }^{1} \mathrm{H}$ NMR $\left(300 \mathrm{MHz}\right.$, DMSO-d $\left.{ }_{6}\right): \delta 9.10$ (s, $1 \mathrm{H}$, $\mathrm{NH}), 7,64(\mathrm{~s}, 1 \mathrm{H}, \mathrm{NH}), 6.83-7.13(\mathrm{~m}, 4 \mathrm{H}, \mathrm{ArH}), 5.07(\mathrm{~s}, 1 \mathrm{H}$, $\mathrm{CH}), 3.96\left(\mathrm{q}, J=7.5 \mathrm{~Hz}, 2 \mathrm{H}, \mathrm{OCH}_{2}\right), 3.69\left(\mathrm{~s}, 3 \mathrm{H}, \mathrm{OCH}_{3}\right), 2.21$ (s, $\left.3 \mathrm{H}, \mathrm{CH}_{3}\right), 1.07$ (t, $\left.J=7.5 \mathrm{~Hz}, 3 \mathrm{H}, \mathrm{OCH}_{2} \mathrm{CH}_{3}\right) ;{ }^{13} \mathrm{C} \mathrm{NMR}(75$ MHz, DMSO-d 6 ): $\delta 165.9,158.9,152.6,148.5,137.5,127.9$, $114.2,100.0,59.6,55.5,53.8,18.2,14.6$.

Ethyl 4-(4-methoxyphenyl)-6-methyl-2-thioxo-1,2,3,4-tetrahydropyrimidine-5 carboxyl -ate (4g). $\mathrm{Mp} 153-155^{\circ} \mathrm{C}$ (lit. [27] $\left.153^{\circ} \mathrm{C}\right) .{ }^{1} \mathrm{H}$ NMR (300 MHz, DMSO-d $\left.{ }_{6}\right): \delta 9.12$ (s, $1 \mathrm{H}$, $\mathrm{NH}), 7.63(\mathrm{~s}, 1 \mathrm{H}, \mathrm{NH}), 6.83-7.14(\mathrm{~m}, 4 \mathrm{H}, \mathrm{ArH}), 5,07$ (s, 1H, $\mathrm{CH}), 3.96$ (q, $\left.J=7.3 \mathrm{~Hz}, 2 \mathrm{H}, \mathrm{OCH}_{2}\right), 3.69\left(\mathrm{~s}, 3 \mathrm{H}, \mathrm{OCH}_{3}\right), 2.21$ (s, $\left.3 \mathrm{H}, \mathrm{CH}_{3}\right), 1.10\left(\mathrm{t}, J=7.2 \mathrm{~Hz}, 3 \mathrm{H}, \mathrm{OCH}_{2} \mathrm{CH}_{3}\right) ;{ }^{13} \mathrm{C} \mathrm{NMR}(75$ MHz, DMSO-d $\left.{ }_{6}\right): \delta 165.9,158.9,152.6,148.5,137.5,137.5$, $127.8,114.2,100.1,59.6,55.5,53.8,18.2,14.5$.

Ethyl 4-(4-(dimethylamino) phenyl)-6-methyl-2-oxo-1,2,3, 4-tetrahydropyrimidine-5-car-boxylate (4h). $\mathrm{Mp} 228-230^{\circ} \mathrm{C}$ (lit.[28] 228-230 ${ }^{\circ} \mathrm{C}$ ). ${ }^{1} \mathrm{H}$ NMR (300 MHz, DMSO-d ${ }_{6}$ ): $\delta 9.06$ (s, $1 \mathrm{H}, \mathrm{NH}), 7.68(\mathrm{~s}, 1 \mathrm{H}, \mathrm{NH}), 6.61-7.56(\mathrm{~m}, 4 \mathrm{H}, \mathrm{ArH}), 5.01(\mathrm{~s}$, $1 \mathrm{H}, \mathrm{CH}), 3.97$ (q, $\left.J=7.3 \mathrm{~Hz}, 2 \mathrm{H}, \mathrm{OCH}_{2}\right), 3.02\left(\mathrm{~s}, 3 \mathrm{H}, \mathrm{NCH}_{3}\right.$ ), $3.02\left(\mathrm{~s}, 3 \mathrm{H}, \mathrm{NCH}_{3}\right), 2.20\left(\mathrm{~s}, 3 \mathrm{H}, \mathrm{CH}_{3}\right), 1.11$ (t, $J=7.3 \mathrm{~Hz}, 3 \mathrm{H}$, $\left.\mathrm{OCH}_{2} \mathrm{CH}_{3}\right) ;{ }^{13} \mathrm{C}$ NMR (75 MHz, DMSO-d $\left.{ }_{6}\right): \delta 190.4,165.9$, $152.8,150.2,147.9,133.0,127.4,112.7,111.5,100.4,59.6$, $53.8,18.2,14.6$.

\section{References}

1. (a) Bienayme, H.; Hulme, C.; Oddon, G.; Schmitt, P. Chem. Eur. J. 2000, 6, 3321-3329. (b) Domling, A.; Ugi, I. Angew. Chem., Int. Ed. 2000, 39, 316.

2. Biginelli, P. Gazz. Chim. Ital. 1893, 23, 360-413.

3. Aslam, M.; Verma, S. Int. J. Chem. Tech Res. 2012, 4, 109-111.

4. Nevagi, R. J.; Narkhede, H. I. Der Pharma Chem. 2014, 6, 135-139.

5. Kumar, P. S.; Idhayadhullal, A.; Abdul-Nasser, A. J.; Selvin, J. J. Serb. Chem. Soc. 2011, 76, 1-11.

6. Lloyd, J.; Finlay, H. J.; Vacarro, W.; Hyunh, T.; Kover, A.; Bhandaru, R.; Yan, L.; Atwal, K.; Conder, M. L.; Jenkins-West, T.; Shi, H.; Huang, C.; Li, D.; Sun, H.; Levesque, P. Bioorg. Med. Chem. Lett. 2010, 20, 1436-1439.

7. (a) Atwal, K. S.; Swanson, B. N.; Unger, S. E.; Floyd, D. M.; Moreland, S.; Hedberg, A.; O'Reilly, B. C. J. Med. Chem. 1991, 34, 806-811. (b) Grover, G. J.; Dzwomczyk, S.; McMullen, D. M.; Normadinam, C. S.; Sleph, P. G.; Moreland, S. J. J. Cardiovasc. Pharmacol. 1995, 26, 289-294. (c) Zorkun, I. S.; Sarac, S.; Celebib, S.; Erolb, K. Bioorg. Med. Chem. 2006, 14, 8582-8589. (d) Sehon, C. A.; Wang, G. Z.; Viet, A. Q.; Goodman, K. B.; 
Dowdell, S. E.; Elkins, P. A.; Semus, S. F.; Evans, C.; Jolivette, L. J.; Kirkpatrick, R. B.; Dul, E.; Khandekar, S. S.; Yi, T.; Wright, L. L.; Smith, G. K.; Behm, D. J.; Bentley, R. J. Med. Chem. 2008, 51, 6631-6634. (e) Chikhale, R. V.; Bhole, R. P.; Khedekar, P. B.; Bhusari, K. P. Eur. J. Med. Chem. 2009, 44, 3645-3653. (f) Alam, O.; Khan, S. A.; Siddiqui, N.; Ahsan, W.; Verma, S. P.; Gilani, S. J. Eur. J. Med. Chem. 2010, 45, 5113-5119.

8. (a) Silder, D. R.; Larsen, R. D.; Chartrain, M.; Ikemote, N.; Roerber, C. M.; Taylor, C. S.; Li, W.; Bills, G. F. PCT Int. Appl. WO 1999, 07695. (b) Kappe, C. O.; Fabian, W. M. F.; Semones, M. A. Tetrahedron 1997, 53, 2803-2816.

9. Bruce, M. A.; Pointdexter, G. S.; Johnson, G. PCT Int. Appl. WO 1998, 33791.

10. (a) Bose, D. S.; Sudharshan, M.; Chavhan, S. W. Arkivoc 2005, iii, 228-236. (b) Hajelsiddig, T. T. H.; Saeed, A. E. M. Int. J. Pharm. Sci. Res. 2015, 6, 2191-2196.

11. Russowsky, D.; Lopes, F. A.; da Silva, V. S. S.; Canto, K. F. S.; Montes D’Oca, M. G.; Godoi, M. N. J. Braz. Chem. Soc. 2004, 15 , 165-169.

12. Reddy, Y. T.; Rajitha, B.; Reddy, P. N.; Kumar, B. S.; Rao, V. P. Synth. Commun. 2004, 34, 3821-3825.

13. Paraskar, A. S.; Dewkar, G. K.; Sudalai, A. Tetrahedron Lett. 2003, 44, 3305-3308.

14. Lu, J.; Bai, Y. Synthesis 2002, 466-470.

15. Yadav, J. S.; Reddy, B. V. S.; Srinivas, R.; Venugopal, C.; Ramalingam, T. Synthesis 2001, 1341-1345.

16. Ma, Y.; Qian, C.; Wang, L.; Yang, M. J. Org. Chem. 2000, 65, 3864-3868.

17. Hu, E. H.; Sidler, D. R.; Dolling, U. H. J. Org. Chem. 1998, 63, 3454-3457.

18. Ranu, B. C.; Hajra, A.; Jana, U. J. Org. Chem. 2000, 65, 62706272.

19. Reddy, C. V.; Mahesh, M.; Raju, P. V. V. K.; Babu, T. R.; Reddy, V. V. N. Tetrahedron Lett. 2002, 43, 2657-2659.

20. Fu, N. Y.; Yuan, Y. F.; Cao, Z.; Wang, S. W.; Wang, J. T.; Peppe, C. Tetrahedron 2002, 58, 4801-4807.

21. Bose, D. S.; Fatima, L.; Mereyala, H. B. J. Org. Chem. 2003, 68, 587-590.

22. Carlos, R. D.; Bernardi, D.; Kirsch, G. Tetrahedron Lett. 2007, 48, 5777-5780.

23. Kappe, C. O.; Kumar, D.; Varma, R. S. Synthesis 1999, 17991803.
24. Salehi, P.; Dabiri, M.; Zolfigol, M. A.; Bodaghi-Fard, M. A. Tetrahedron Lett. 2003, 44, 2889-2891.

25 Sowmiya, M.; Sharma, A.; Parsodkar, S.; Mishra, B. G.; Dubey, A. Appl. Catal. A, 2007, 333, 272-280.

26. Ahn, B. J.; Gang, M. S.; Chae, K.; Oh, Y.; Shin, J.; Chang, W. A. J. Ind. Eng. Chem. 2008, 14, 401-405.

27. Debache, A.; Boumoud, B.; Amimour, M.; Belfaitah, A.; Rhouati, S.; Carboni, B. Tetrahedron Lett. 2006, 47, 5697-5699.

28. Liu, C. J.; Wang, J. D. Molecules 2009, 14, 763-770

29. Nasr-Esfahani, M.; Hoseini, S. J.; Mohammadi, F. Chin. J. Catal. 2011, 32, 1484-1489.

30. Moghaddas, M.; Davoodnia, A.; Heravi, M. M.; Tavakoli-Hoseini, N. Chin. J. Catal. 2012, 33, 706-710.

31. Herbst, R. M.; Shemin, D. Org. Syn. Coll. 1943, 2, 11.

32. Muhammad Y.; Marek B.; Lubna S.; Shahzadd, S. A.; Ahmede, M.; Ashraff, M.; Alamf, U.; Khang, I. U.; Khan, A. F. Bioorg. Chem. 2014, 54, 96-104.

33. Kang, S.; Coopera, G.; Dunned, S. F.; Luand, C. H.; Surmeier, J. D., Silverman, R. B. Bioorg. Med. Chem. 2013, 21, 4365-4373.

34. Jetti, S. R.; Verma, D.; Jain, S. Arab. J. Chem. 2014, 689-701.

35. Starcevich, J. T.; Laughlin, J. T.; Mohan, R. S. Tetrahedron Lett. 2013, 54, 983-985.

36. Heidarizadeh, F.; Nezhad, E. R.; Sajjadifar, S. Scientia Iranica $C$ 2013, 20, 561-565.

37. Keivanloo, A.; Mirzaee, M.; Bakherad, M.; Soozani, A. Chin. J. Catal. 2014, 35 362-367.

38. Safari, J., Ravandi, S. G. J. Mol. Struct. 2014, 241, 1065-1066.

39. Tayebee, R.; Amini, M. M.; Ghadamgahi, M.; Armaghan, M. $J$. Mol. Catal. A 2013, 366, 266-274.

40. Benzekri, Z.; El Mejdoub, K.; Boukhris, S.; Sallek, B.; Lakhrissi, B.; Souizi, A. Synth. Commun. 2016, 46, 442-452.

41. An, L.; Han, L.; Wang, Z.; Huang, T.; Zhu, H. Biol. Pharm. Bull. 2016, 39, 267-271.

42. S. Girase, P.; J. Khairnar, B.; V. Nagarale, D.; R. Chaudhari, B. Der Pharma Chem. 2015, 7, 241-247.

43. Gopinath, K. R.; Premkumar, H. B.; Shekar, H. S.; Rajendraprasad, K. J.; Nagabhushana, H.; Krishnappa, M. World J. Pharm. Pharmaceut. Sci. 2016, 5, 1578-1589.

44. Azimi, S.; Hariri, M. Iran. Chem. Commun. 2015, 3, 13-20

45. Zare, A.; Nasouri, Z. J. Mol. Liq. 2016, 216, 364-369.

46. Bashti, A.; Kiasat, A. R. Org. Chem. Res. 2016, 2, 28-38. 